\title{
Sex hormones modulate brain damage in multiple sclerosis: MRI evidence
}

\author{
V Tomassini, E Onesti, C Mainero, E Giugni, A Paolillo, M Salvetti, F Nicoletti, C Pozzilli
}

J Neurol Neurosurg Psychiatry 2005;76:272-275. doi: 10.1136/jnnp.2003.033324

Background: Sex related differences in the course and severity of multiple sclerosis (MS) could be mediated by the sex hormones.

Objective: To investigate the relation between serum sex hormone concentrations and characteristics of tissue damage on conventional magnetic resonance imaging (MRI) in men and women suffering from relapsing-remitting MS.

Results: Serum testosterone was significantly lower in women with MS than in controls. The lowest levels were found in women with a greater number of gadolinium enhancing lesions. A positive correlation was observed between testosterone concentrations and both tissue damage on MRI and clinical disability. In men, there was a positive correlation between oestradiol concentrations and brain damage.

Conclusions: The hormone related modulation of pathological changes supports the hypothesis that sex hormones play a role in the inflammation, damage, and repair mechanisms typical of MS.

S everal lines of evidence suggest that gender affects the clinical course of multiple sclerosis (MS), male sex being associated with a more progressive and severe outcome than female sex. ${ }^{1-3}$ Experimental data in animal models of MS have confirmed a sex related difference in the susceptibility, course, and severity of the disease. ${ }^{4}$ They have hinted at the fact that vulnerability of the central nervous system (CNS) to damage, as well as the spontaneous recovery, might vary between men and women. ${ }^{5-7}$

Using magnetic resonance imaging (MRI), it has previously been shown that men with MS are less prone to inflammatory lesions but more susceptible to destructive lesions than women. ${ }^{89}$ An association between sex hormone concentrations and variations in disease activity as assessed by enhanced MRI has been also reported..$^{10}$

The mechanisms by which sex hormones might affect brain damage in MS vary from innate susceptibility to sex related differences in the development of the inflammatory process, limitation of tissue injury, or the repair mechanisms.

Finding an effect of sex hormones on the dynamics of the MS disease process would support the hypothesis that sex hormones play a specific role in brain damage and repair mechanisms typical of MS. In order to clarify whether sex hormones mediate the difference in the MRI characteristics of brain damage, we investigated the relation between serum concentrations of sex hormones and MRI features in a population of subjects suffering from relapsing-remitting MS (RRMS).

\section{METHODS}

\section{Patients}

We included 60 RRMS patients ( 35 women and 25 men) with a mean (SD) age of 32.3 (7.6) years, a disease duration of 6.2
(5.4) years (range 1 to 26), and a median (range) expanded disability status scale (EDSS) score of 1.5 ( 1 to 5.5 ). The subjects had no history of disease modifying treatments and had not experienced relapses or received steroid treatment in the previous two months. Female patients had normal menstrual cycles and had never employed oral contraceptives or undergone hormone replacement therapy. Thirty six healthy, age matched subjects served as controls.

\section{Study protocol}

Serum follicle stimulating hormone $(\mathrm{FSH})(\mathrm{mU} / \mathrm{ml})$, luteinising hormone $(\mathrm{LH})(\mathrm{mU} / \mathrm{ml}), 17-\beta$ oestradiol $(\mathrm{pg} / \mathrm{ml})$, testosterone $(\mathrm{ng} / \mathrm{ml})$, and dehydroepiandrosterone sulphate (DHEAs) $(\mu \mathrm{g} / \mathrm{ml})$ were determined in both men and women. In women these hormones were measured during the follicular (third to ninth day) and luteal phases (21st to 28 th day) of their menstrual cycle, once at each point, while progesterone $(\mathrm{ng} / \mathrm{ml})$ was quantified during the luteal phase.

In parallel, brain MRI was carried out using a $1.5 \mathrm{~T}$ superconductive system (Philips Gyroscan NT 15, Erlangen, Germany). The following sequences were obtained:

- conventional dual spin echo: proton density (PD) and T2 weighted images (T2WI): time of repetition $(\mathrm{TR})=2000$ $\mathrm{ms}$; time of echo $(\mathrm{TE})=20 / 90 \mathrm{~ms}$;

- pre- and post-contrast Tl weighted images (TIWI): $\mathrm{TR}=550 \mathrm{~ms} ; \mathrm{TE}=12 \mathrm{~ms}$; single $0.1 \mathrm{mmol} / \mathrm{kg}$ dose of $\mathrm{Gd}$ DTPA (gadolinium diethylenetetraminepenta-acetic acid).

For each sequence, axial contiguous slices with $3 \mathrm{~mm}$ thickness, $25 \mathrm{~cm}$ field of view, and $256 \times 256$ matrix were acquired.

\section{MRI analysis}

All MRI scans were archived onto electronic media and transferred to a SUN workstation (Sun Microsystems, Palo Alto, California, USA). For each patient, we determined the number of enhancing lesions as well as the volume of T2 hyperintense (T2-LL) and T1 hypointense (T1-LL) lesions. T1LL measures the volume of "black holes", which are lesions with lower signal intensity than surrounding white matter but equal to or lower than grey matter. T2-LL and T1-LL were assessed using a semiautomated contouring technique (Dispim, Dispimage). ${ }^{12}{ }^{13}$ The quantification of black holes on post-Gd scans followed standard practice, and was justifiable as many low signal intensity lesions on pre-Gd Tl weighted images were acute lesions that enhanced following $\mathrm{Gd}$ administration.

\section{Statistical methods}

Differences in serum sex hormone concentrations between MS patients and controls and among MS subgroups were

Abbreviations: DHEAs, dehydroepiandrosterone sulphate; EDSS, expanded disability status scale; FSH, follicle stimulating hormone; Gd, gadolinium; LH, luteinising hormone; RRMS, relapsing-remitting multiple sclerosis 
Table 1 Serum sex hormone concentrations in patients with multiple sclerosis and controls

\begin{tabular}{|c|c|c|c|c|c|c|c|c|c|}
\hline & \multicolumn{6}{|l|}{ Women } & & & \\
\hline & \multicolumn{3}{|c|}{ Follicular phase } & \multicolumn{3}{|l|}{ Luteal phase } & \multicolumn{3}{|l|}{ Men } \\
\hline & $\begin{array}{l}\text { MS } \\
(n=35)\end{array}$ & $\begin{array}{l}\text { Controls } \\
(n=18)\end{array}$ & p Value* & $\begin{array}{l}\text { MS } \\
(n=35)\end{array}$ & $\begin{array}{l}\text { Controls } \\
(n=18)\end{array}$ & p Value* & $\begin{array}{l}\text { MS } \\
(n=25)\end{array}$ & $\begin{array}{l}\text { Controls } \\
(n=18)\end{array}$ & p Value* \\
\hline FSH & $5.61(3.7)$ & $8.29(7.2)$ & 0.08 & $3.42(2.7)$ & $3.92(2.0)$ & 0.49 & $4.65(1.6)$ & $6.23(3.5)$ & 0.06 \\
\hline LH & $5.03(2.1)$ & $5.53(3.1)$ & 0.50 & $4.52(9.4)$ & $6.60(7.9)$ & 0.42 & $4.51(2.7)$ & $4.49(1.8)$ & 0.99 \\
\hline Oestradiol & $82.54(87.1)$ & $56.22(44.5)$ & 0.24 & $80.17(52.4)$ & $150.94(165.6)$ & 0.02 & 41.40 (13.2) & $47.50(15.8)$ & 0.18 \\
\hline Testosterone & $0.49(0.1)$ & $0.58(0.1)$ & 0.03 & $0.47(0.1)$ & $0.63(0.1)$ & 0.0001 & $3.68(1.0)$ & $4.08(1.0)$ & 0.20 \\
\hline DHEAs & $2.06(1.2)$ & $2.61(1.4)$ & 0.14 & $2.01(1.2)$ & $2.48(1.0)$ & 0.16 & $2.63(1.0)$ & $2.41(0.8)$ & 0.43 \\
\hline Progesterone & $0.66(0.2)$ & $0.82(0.3)$ & 0.04 & $6.39(7.6)$ & $7.03(7.0)$ & 0.77 & - & - & - \\
\hline
\end{tabular}

compared using the unpaired Student's $t$ test. The MannWhitney U test was used to compare MRI derived measures in male and female patients. In both sexes, the relations between MRI derived data and sex hormone concentrations were evaluated by the Spearman rank correlation coefficient.

\section{RESULTS}

There were no significant differences between women and men in disease duration (mean (SD), women $v$ men: 6.08 (4.9) $v 6.14(5.7)$, NS) or EDSS (women $v$ men: $2.0(1.3) v$ 2.08 (0.9), NS). The mean number of Gd enhancing lesions was significantly greater in women than in men (women $v$ men: $3.4(5.0) v 1.1(2.5) ; \mathrm{p}=0.004)$. However, the extent of brain damage was similar in both groups, as measured by $\mathrm{T} 2$ LL (women $v$ men: $10.92(8.2) \mathrm{cm}^{3} v 10.78(8.3) \mathrm{cm}^{3}, \mathrm{NS}$ ) and Tl-LL (women $v$ men: $13.17(0.2) \mathrm{cm}^{3} v 15.05(1.6) \mathrm{cm}^{3}, \mathrm{NS}$ ).

In women, no significant difference was observed in the frequency of enhancing lesions, T2-LL, or T1-LL between scans obtained during the follicular and luteal phases.

Mean serum hormone concentrations in MS patients and controls are shown in table 1 . No significant difference in sex hormone levels was noted between male patients and controls. Women with MS had lower testosterone concentrations than controls in the follicular as well as in the luteal phase of the menstrual cycle.

Considering data on the testosterone levels of healthy women, a normal score for testosterone (within 2 SD of the mean) was calculated. We used this score as a cut off value to identify women with abnormally low testosterone concentrations (more than 2 SD below the mean for the controls). A significantly greater number of Gd enhancing lesions was found in the seven women with abnormally low testosterone levels compared with those with a normal testosterone (7.4 v $2.5, \mathrm{p}=0.02$ )
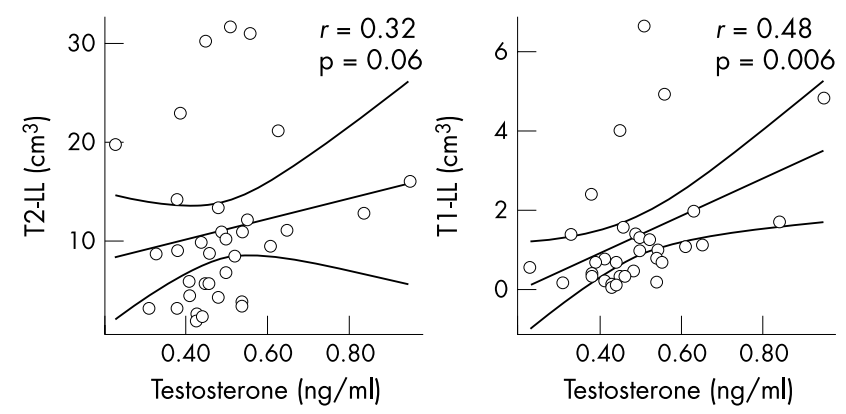

To analyse the relation between MRI derived measures and the concentrations of each hormone in women, only the MRI scan acquired during the follicular phase was considered. Higher serum testosterone levels in women with MS were associated with higher Tl-LL $(r=0.48, \mathrm{p}=0.006)$, but not with T2-LL $(r=0.32, \mathrm{p}=0.06)$. No significant relation was found between MRI data and oestradiol concentrations (T2-LL: $r=-0.22, \mathrm{p}=0.21$; T1-LL: $r=-0.17, \mathrm{p}=0.32$ ) (fig 1).

In men, a positive correlation was found between oestradiol concentrations and both T2-LL $(r=0.47$, $\mathrm{p}=0.02)$ and Tl-LL $(r=0.43, \mathrm{p}=0.04)$. No significant relation was shown between MRI findings and testosterone concentrations (T2-LL: $r=-0.08 ; \mathrm{p}=0.93$; T1-LL: $r=0.12$, $\mathrm{p}=0.57$ ) (fig 2). Furthermore, no significant correlations were found between the other sex hormone levels and MRI measures of tissue damage.

In women, a trend towards significance was found in the relation between testosterone concentrations and neurological disability as measured by the EDSS $(r=0.29, \mathrm{p}=0.05)$. In men, EDSS and oestradiol levels were not significantly associated $(r=-0.15, \mathrm{p}=0.65)$.

\section{DISCUSSION}

Several studies have suggested that gender affects the clinical course of MS. ${ }^{1-3}$ Using MRI in a large cohort of patients, we have previously shown that there is a pathological counterpart for the different clinical course, with women having more inflammatory but less destructive lesions than men. ${ }^{9}$ Results from this study partially supported previous findings (that is, a greater number of Gd enhancing lesions in women than in men), but failed to detect any difference in the proportion of lesions evolving into "black holes", probably because of the small sample size.

Figure 1 Relation between sex hormone levels and magnetic resonance imaging (MRI) findings in women with multiple sclerosis. Testosterone concentrations were significantly associated with T1-LL $(r=0.48, p=0.006)$, but not with T2-LL $(r=0.32, p=0.06)$. No significant correlation was observed between MRI findings and oestradiol levels (T2-LL: $r=-0.22 ; p=0.21 ; \mathrm{Tl}-\mathrm{LL}: r=-0.17, p=0.32$ ). T1-LL, T1 hypointense lesions; T2-LL, T2 hyperintense lesions. 

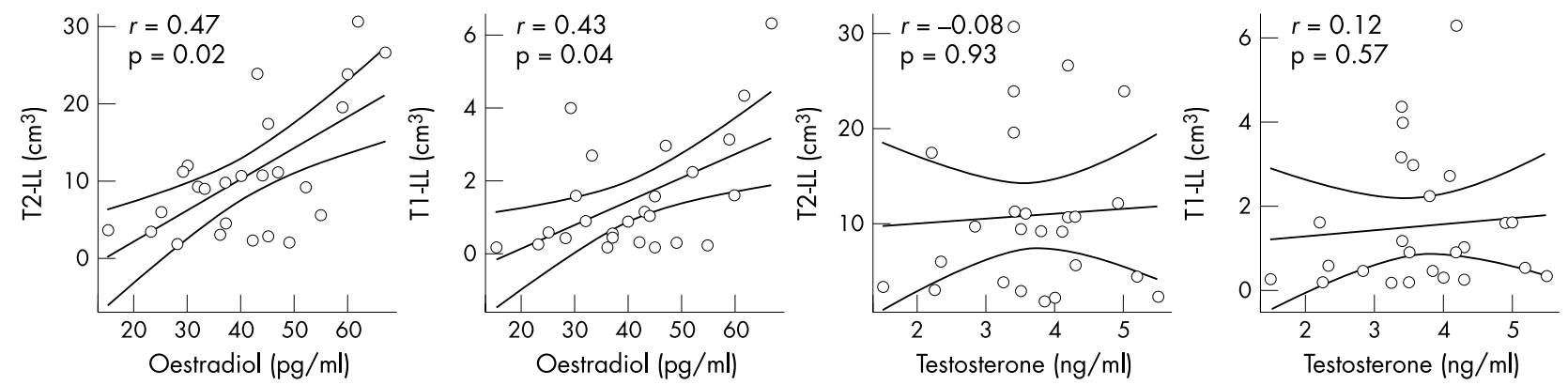

Figure 2 Relation between sex hormone levels and magnetic resonance imaging (MRI) findings in men with multiple sclerosis. Oestradiol concentrations correlated with both T2-LL $(r=0.47, \mathrm{p}=0.02)$ and T1-LL $(r=0.43, \mathrm{p}=0.04)$. No significant association was found between MRI findings and testosterone levels (T2-LL: $r=-0.08 ; p=0.93 ; \mathrm{T} 1-\mathrm{LL}: r=0.12, p=0.57$ ). T1-LL, T1 hypointense lesions; T2-LL, T2 hyperintense lesions.

The primary objective of this study was to determine whether there is a link between the profile of sex hormones and MRI characteristics of brain damage in RRMS. As a first step, we investigated whether MS patients had hormonal patterns that were different from those in healthy controls. The results showed that women with MS had lower testosterone concentrations than normal subjects. Women with hormonal levels more than 2 SD below those of healthy controls had more enhancing lesions than women with normal testosterone concentrations. In autoimmune diseases other than MS, such as systemic lupus erythematosus and rheumatoid arthritis, where the hormonal balance seems to play a pathophysiological role, ${ }^{14}$ serum testosterone in women was at its lowest level during exacerbations of the disease. ${ }^{15}{ }^{16}$ By contrast, we found no significant differences in the sex hormone levels between men suffering from MS and healthy men. Although the small number of controls compared with patients might have obscured a difference, it can be argued that testosterone concentration was in the normal range in men with MS because they were less prone to develop inflammatory lesions, as has been found previously. ${ }^{9}$ In a study assessing endocrine function in MS, Wei and Lightman ${ }^{17}$ reported an overactivation of the hypothalamic-pituitary-adrenal axis in parallel with inflammation. Furthermore, Foster et al ${ }^{18}$ suggested that inflammatory cytokines might stimulate the hypothalamic-pituitary-gonadal axis resulting in a decreased production of testosterone. The fact that lower testosterone levels could also account for lower levels of oestradiol during the luteal phase cannot be excluded as, in the ovarian steroidogenesis, 17- $\beta$ oestradiol is produced from testosterone in the granulosa cells. Lower luteal oestradiol levels cannot be explained by an abnormality in the menstrual cycle (such as anovulation), as progesterone levels were augmented during the luteal phase in a similar way to the control population.

Moreover, in women affected by MS, higher testosterone concentrations were significantly associated with the likelihood of irreversible tissue injury (that is, Tl-LL) and clinical disability, whereas in men with MS, there was a positive correlation between serum oestradiol and both T2-LL and T1LL. Hyperintense lesions on T2 weighted images provided a non-specific measure of various pathological substrates, ranging from oedema and inflammation to irreversible demyelination and axonal loss, thus predicting different clinical outcomes. Tl hypointense lesions ("black holes") reflected axonal $\operatorname{loss}^{19}$ and were strongly correlated with neurological disability in $\mathrm{MS} .{ }^{20}$ On the basis of the relation between serum levels of sex hormones and MRI features, it can be hypothesised that, in RRMS, both oestradiol and testosterone were implicated in the tissue response to brain damage. Our study did not directly address causality, so that determining whether increases in serum hormone levels reflected the brain's compensatory repair mechanisms during injury or if these molecules actually contribute to the development of tissue damage was not possible. In a model of acute brain harm, such as that produced by head trauma, increases in endogenous sex hormones have been shown to have a protective effect on brain tissue. ${ }^{21} 22$ There is also evidence that testosterone and $17-\beta$ oestradiol downregulate reactive gliosis and astrocyte proliferation, ${ }^{23}$ the major obstacles to axonal regeneration in the mammalian $\mathrm{CNS}^{24}$ On the other hand, testosterone has been shown to render oligodendrocytes more vulnerable to excitotoxic damage..$^{25}$

The differential effect of the sex hormones on brain damage observed in our study (that is, testosterone in women and oestradiol in men) may, at least in part, be explained by experimental data. In male rats, the administration of 17- $\beta$ oestradiol has been shown to improve both biochemical and neurological outcomes following traumatic brain injury. In contrast, the administration of this steroid hormone to female rats appears to exacerbate biochemical changes in the acute phase following traumatic brain damage. ${ }^{26}$ Emerson et $a l^{26}$ have suggested that oestrogen treatment has both receptor mediated and receptor independent effects on cellular energy metabolism. The deleterious effects noted in women but not in men probably reflect the fact that the female brain has a much greater receptor binding capacity (up to 100\%) than the male brain, and consequently is at greater risk of receptor mediated excitotoxicity and cell death. ${ }^{27}$

\section{Conclusions}

Our study indicates that serum testosterone is reduced in women suffering from MS, as in other autoimmune inflammatory syndromes, especially during the active phase of the disease, as documented by brain MRI. We therefore propose that oestrogens and testosterone play a role in modulating the development of brain tissue damage in MS. The respective contribution of these two hormones and their types of actions and interactions deserve further analysis.
Authors' affiliations
V Tomassini, E Onesti, C Mainero, E Giugni, A Paolillo, M Salvetti, C Pozzilli, Department of Neurological Sciences, "La Sapienza" University, Rome, Italy
F Nicoletti, Department of Pharmacology, "La Sapienza" University, Rome, and INM Neuromed, Pozzilli, Italy
Competing interests: none declared
Correspondence to: Professor Carlo Pozzilli, Department of Neurological Sciences, II Faculty of Medicine, University "La Sapienza", viale dell'Università 30, 00185 Rome, Italy; carlo.pozzilli@uniroma1.it 
Received 26 November 2003

In revised form 5 May 2004

Accepted 11 June 2004

\section{REFERENCES}

1 Weinshenker BG, Bass B, Rice GP, et al. The natural history of multiple sclerosis: a geographically based study. 2. Predictive value of the early clinical course. Brain 1989:112:1419-28.

2 Hawkins SA, McDonnell GV. Benign multiple sclerosis? Clinical course, long term follow up, and assessment of prognostic factors. J Neurol Neurosurg Psychiatry 1999;67:148-52.

3 Confavreux C, Vukusic S, Adeleine P. Early clinical predictors and progression of irreversible disability in multiple sclerosis: an amnesic process. Brain 2003; 126:770-82

4 Voskuhl RR. Gender issues and multiple sclerosis. Curr Neurol Neurosci Rep 2002;2:277-86.

5 Hall ED, Pazara KE, Linseman KL. Sex differences in postischemic neuronal necrosis in gerbils. J Cereb Blood Flow Metab 1991;11:292-8.

6 Alley J, Khasabov S, Simone D, et al. More severe neurologic deficits in SJ/J male than female mice following Theiler's virus-induced CNS demyelination. Exp Neurol 2003;180:14-24.

7 Hauben E, Mizrahi T, Agranov E, et al. Sexual dimorphism in the spontaneous recovery from spinal cord injury: a gender gap in beneficial autoimmunity? Eur J Neurosci 2002;16:1731-40.

8 Weatherby SJ, Mann CL, Davies MB, et al. A pilot study of the relationship between gadolinium-enhancing lesions, gender effect and polymorphisms of antioxidant enzymes in multiple sclerosis. J Neurol 2000;247:467-70.

9 Pozzilli C, Tomassini V, Marinelli F, et al. "Gender gap" in multiple sclerosis: magnetic resonance imaging evidence. Eur J Neurol 2003;10:95-7.

10 Pozzilli C, Falaschi P, Mainero C, et al. MRI in multiple sclerosis during the menstrual cycle: relationship with sex hormone patterns. Neurology 1999:53:622-4.

11 Bansil S, Lee HJ, Jindal S, et al. Correlation between sex hormones and magnetic resonance imaging lesions in multiple sclerosis. Acta Neurol Scand 1999:99:91-4

12 Grimaud J, Lai M, Thorpe J, et al. Quantification of MRI lesion load in multiple sclerosis: a comparison of three computer-assisted techniques. Magn Reson Imaging 1996;14:495-505.

13 Miller DH, Grossman RI, Reingold SC, et al. The role of magnetic resonance techniques in understanding and managing multiple sclerosis. Brain 1998;121:3-24.
14 Wilder RL. Neuroimmunoendocrinology of the rheumatic diseases: past, present, and future. Ann NY Acad Sci 2002;966:13-19.

15 Lahita RG, Bradlow HL, Ginzler E, et al. Low plasma androgens in women with systemic lupus erythematosus. Arthritis Rheum 1987:30:241-8.

16 Kanik KS, Chrousos GP, Schumacher HR, et al. Adrenocorticotropin, glucocorticoid, and androgen secretion in patients with new onset synovitis/ rheumatoid arthritis: relations with indices of inflammation. J Clin Endocrinol Metab 2000;85:1461-6.

17 Wei T, Lightman SL. The neuroendocrine axis in patients with multiple sclerosis. Brain 1997; 120:1067-76.

18 Foster SC, Daniels C, Bourdette DN, et al. Dysregulation of the hypothalamic-pituitary-gonadal axis in experimental autoimmune encephalomyelitis and multiple sclerosis. J Neuroimmunol 2003; 140:78-87.

19 van Walderveen MA, Kamphorst W, Scheltens P, et al. Histopathologic correlate of hypointense lesions on T1-weighted spin-echo MRI in multiple sclerosis. Neurology 1998;50:1282-8.

20 Truyen $\mathrm{L}$, van Waesberghe $\mathrm{JH}$, van Walderveen MA, et al. Accumulation of hypointense lesions ("black holes") on T1 spin-echo MRI correlates with disease progression in multiple sclerosis. Neurology 1996;47:1469-76.

21 Roof RL, Hall, eds. Gender differences in acute CNS trauma and stroke: neuroprotective effects of estrogen and progesterone. J Neurotrauma 2000;17:367-88

22 Bramlett HM, Dietrich WD. Neuropathological protection after traumatic brain injury in intact female rats versus males or ovariectomized females. J Neurotrauma 2001;18:891-900.

23 Garcia-Estrada J, Del Rio JA, Luquin S, et al. Gonadal hormones downregulate reactive gliosis and astrocyte proliferation after a penetrating brain injury. Brain Res 1993;628:271-8

24 Bovolenta P, Wandosell F, Nieto-Sampedro M. CNS glial scar tissue: a source of molecules which inhibit central neurite outgrowth. Prog Brain Res 1992;94:367-79.

25 Caruso A, Di Giorgi Gerevini V, Castiglione M, et al. Testosterone amplifies excitotoxic damage of cultured oligodendrocytes. J Neurochem 2004;88: 1179-85.

26 Emerson CS, Headrick JP, Vink R. Estrogen improves biochemical and neurologic outcome following traumatic brain injury in male rats, but not in females. Brain Res 1993;608:95-100.

27 Brown TJ, Hochberg RB, Zielinski JE, et al. Regional sex differences in cell nuclear estrogen-binding capacity in the rat hypothalamus and preoptic area Endocrinology 1988;123:1761-70. 